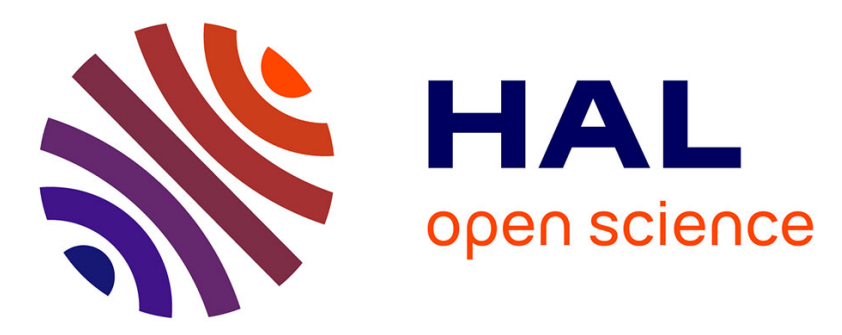

\title{
A Formalization of Grassmann-Cayley Algebra in COQ and Its Application to Theorem Proving in Projective Geometry
}

\author{
Laurent Fuchs, Laurent Thery
}

\section{- To cite this version:}

Laurent Fuchs, Laurent Thery. A Formalization of Grassmann-Cayley Algebra in COQ and Its Application to Theorem Proving in Projective Geometry. Automated Deduction in Geometry, ADG 2010, Jul 2010, Munich, Germany. pp.51-62, 10.1007/978-3-642-25070-5_3 . hal-00657901

\section{HAL Id: hal-00657901 \\ https://hal.science/hal-00657901}

Submitted on 9 Jan 2012

HAL is a multi-disciplinary open access archive for the deposit and dissemination of scientific research documents, whether they are published or not. The documents may come from teaching and research institutions in France or abroad, or from public or private research centers.
L'archive ouverte pluridisciplinaire HAL, est destinée au dépôt et à la diffusion de documents scientifiques de niveau recherche, publiés ou non, émanant des établissements d'enseignement et de recherche français ou étrangers, des laboratoires publics ou privés. 


\title{
A Formalization of Grassmann-Cayley Algebra in COQ and its Application to Theorem Proving in Projective Geometry ${ }^{\star}$
}

\author{
Laurent Fuchs $^{1} \quad$ Laurent Théry ${ }^{2}$ \\ 1 XLIM-SIC UMR CNRS 6172 - Poitiers University, France, \\ Laurent.Fuchs@sic.univ-poitiers.fr \\ 2 INRIA Sophia Antipolis - Méditerranée, France, \\ Laurent.Thery@inria.fr
}

\begin{abstract}
This paper presents a formalization of Grassmann-Cayley algebra 6] that has been done in the CoQ 2] proof assistant. The formalization is based on a data structure that represents elements of the algebra as complete binary trees. This allows to define the algebra products recursively. Using this formalization, published proofs of Pappus' and Desargues' theorem [7] are interactively derived. A method that automatically proves projective geometric theorems [11] is also translated successfully into the proposed formalization.
\end{abstract}

\section{Introduction}

A well-known application of Grassmann-Cayley algebra is automated theorem proving in projective geometry (see for example [14/10]). The usual method is to translate incidence statements of projective geometry into Grassmann-Cayley expressions. These expressions are then translated into bracket polynomials (i.e. the ring of projective invariants [17]). Finally, the bracket polynomial is factorised to get back an equivalent expression in Grassmann-Cayley algebra.

Our motivation in using a proof assistant such as CoQ [2] is to capture in a single system all the various aspects of Grassmann-Cayley algebra: we want an abstract generic model on which we can not only reason but also perform both numerical and symbolical evaluations.

Hence, our formalization lets us not only formally check the manipulations of expressions within Grassmann-Cayley algebra but also compute with these very same expressions. De facto, it makes explicit the link between the abstract mathematical object and its applications. In the CoQ proof assistant, proofs can be conducted interactively step by step or, using programmed tactics, the expressions can be reduced in a systematic manner. Note that, in our setting, most proofs are parametrized by the dimension of the algebra. So, our development is generic.

Once the formalization of the Grassmann-Cayley algebra is achieved, two kinds of proofs are considered. First some proofs are conducted interactively

\footnotetext{
* This work has been supported by the ANR Galapagos
} 
following step-by-step what can be found in the literature, such as the proof of the Pappus' theorem in [7] or the proofs of Desargues' theorem in [1]. The second kind of proofs are conducted automatically following a method published in [11]. All the examples proposed in [11] have been tested successfully.

In future work, we also plan to connect our formalization with other approaches of incidence geometry, such as those based over ranks [12]13]14. So, our work can be seen as a first step in the study of the formal correctness of automated proof methods in incidence geometry.

This paper is organized as follows. Section 2 introduces Grassmann-Cayley algebra and our choices for the formalization. Section 3 explains how the GrassmannCayley is formalized, how the algebra elements are represented and how the products are defined. Section 4 describes how the formalization can be use to prove theorems of incidence geometry, interactively and automatically.

\section{Formal Grassmann-Cayley Algebra}

Usually, in the literature, the products (join and meet) of the Grassmann-Cayley algebra are introduced by given equations defining their properties. So, they could have been defined in COQ using such an axiomatic approach. However, the main drawback of doing so is that we completely lose the computational aspect of this algebra. In particular, the axiomatic approach gives no hint of how the algebra could actually be implemented on a computer.

For this reason, we favor the definitional approach where the algebra operations are defined as recursive functions over the dimension of the algebra. First, we define a model, i.e. a data-structure that represents elements of the algebra. Then, on this model, we define the usual algebra operations (the join product, the meet product and the duality) and prove that they fulfill the axioms that are used to defined them in the literature. As this representation is quite unusual, we spend some time to detail our data-structure and the related operations.

\subsection{The underlying vector space}

The Grassmann-Cayley algebra $G_{n}$ is defined by adding a second product, the meet product, to the Grassmann algebra (or exterior algebra) of a vector space of dimension $n, V$, over a field $K$ 6]1 where the join product (or the exterior product) is defined.

In order to have a concrete representation of the vectors of $V$, we need to represent them as $n$-tuples of $K^{n}$. This imposes a basis for $V$, say the canonical basis $e_{n}^{i}=\left(\delta_{i, 0}, \ldots, \delta_{i, i}, \ldots, \delta_{i, n-1}\right)$ where $i=0, \ldots, n-1$ and $\delta_{i, j}$ is the Kronecker symbol. Then $V$ is seen as the set of $n$-tuples, $K^{n}$.

As we will see this choice also induces the definition of a basis for $G_{n}$ and this leads to an important change of view in the presentation of the algebra compared to the usual coordinate-free presentation. The elements are represented via their coordinates. 
However, in the Coq proof assistant, this does not force us to deal only with numerical computations. As all the axiomatic properties of the algebra operations are proved, we can also reason symbolically using the coordinatefree presentation. Hence, we obtain an abstract generic model on which both numerical and symbolical evaluations can be performed.

\subsection{The join product}

The first step is to define the join product denoted by $\vee$. It is an associative antisymmetric bilinear product and it can be defined axiomatically by:

$$
\begin{array}{rlrl}
a \vee a & =0 & \lambda a \vee b & =\lambda(a \vee b) \\
b \vee a & =-a \vee b & (a+b) \vee c=a \vee c+b \vee c
\end{array}
$$

where $a$ and $b$ are vectors of $K^{n}$.

The join product $a \vee b$ of two vectors $a$ and $b$ is non-zero if and only if $a$ and $b$ are linearly independent. If $a \vee b$ is non-zero, it is a grade 2 element of the algebra. More generally, if $\left\{a_{1}, \ldots, a_{k}\right\}$ are linearly independent vectors of $K^{n}$ then $a_{1} \vee \cdots \vee a_{k}$ is an element of grade $k$. Such elements, that are join product of vectors, are called extensors or decomposable $k$-vectors. Not all elements of grade $k$ are extensors, they could be linear combination of extensors. In that case they are called homogeneous vectors or $k$-vectors. Elements that are linear combination of elements with different grades are the general elements of the algebra. They are called multi-vectors.

On the basis elements $\left\{e_{n}^{0}, \ldots, e_{n}^{n-1}\right\}$ of $K^{n}$, the join product has the following two behaviors:

$$
\mathrm{e}_{n}^{i} \vee \mathrm{e}_{n}^{i}=0 \quad \quad \mathrm{e}_{n}^{i} \vee \mathrm{e}_{n}^{j}=-\mathrm{e}_{n}^{j} \vee \mathrm{e}_{n}^{i} .
$$

This gives the graded structure of $G_{n}$. The join product of $k$ basis elements generates the subspace of grade $k$ homogeneous elements. Considering $G_{3}$, this means that:

$\{1\}$ generates the elements of grade 0 .

$\left\{\mathrm{e}_{3}^{0}, \mathrm{e}_{3}^{1}, \mathrm{e}_{3}^{2}\right\}$ generates the elements of grade 1 .

$\left\{\mathrm{e}_{3}^{0} \vee \mathrm{e}_{3}^{1}, \mathrm{e}_{3}^{0} \vee \mathrm{e}_{3}^{2}, \mathrm{e}_{3}^{1} \vee \mathrm{e}_{3}^{2}\right\}$ generates the elements of grade 2 .

$\left\{\mathrm{e}_{3}^{0} \vee \mathrm{e}_{3}^{1} \vee \mathrm{e}_{3}^{2}\right\}$ generates the elements of grade 3 .

Hence, $G_{n}$ can be seen as a vector space of dimension $2^{n}$. Our model is a representation of this vector space that allows a computational definition of the products of the Grassmann-Cayley algebra.

\subsection{The meet product}

Retrieving the bracket. Usual presentation of the Grassmann-Cayley algebra 6[118 defines a bracket over the vector space $V$. Given $n$ vectors $a_{1}, \ldots, a_{n}$ the bracket $\left[a_{1}, \ldots, a_{n}\right]$ is a non-degenerate multilinear alternating $n$-form, taking its values into the field $K$. 
The use of the canonical basis of the vector space $K^{n}$ defines a bracket implicitly. The set of elements of grade $n$ generated by $e_{n}^{0} \vee \cdots \vee e_{n}^{n-1}$ is isomorphic to the set of elements of grade 0 via the linear map defined by $i\left(e_{n}^{0} \vee \cdots \vee e_{n}^{n-1}\right)=1$. This linear map defines a non-degenerate multilinear $n$-form over the vectors of $K^{n}$ that is actually a determinant.

Hence, the choice of the canonical basis defines a bracket. This allows us to retrieve the usual definition of the Grassmann-Cayley algebra. This link is used in section 4.2 to introduce automated proof techniques into our formalization.

The Hodge star. Moreover, as $i\left(e_{n}^{0} \vee \cdots \vee e_{n}^{n-1}\right)=\left[e_{n}^{0}, \ldots, e_{n}^{n-1}\right]=1$, the canonical basis is said to be unimodular [1. Then, the Hodge star defined as follows:

$$
*\left(e_{n}^{\rho(0)} \vee_{n} \ldots \vee_{n} e_{n}^{\rho(i)}\right)=e_{n}^{\rho(i+1)} \vee_{n} \ldots \vee_{n} e_{n}^{\rho(n-1)}
$$

where $\rho$ is an even permutation, satisfies the following properties (see [1]):

(i) * maps extensors of grade $k$ to extensors of grade $n-k$,

(ii) $*(1)=e_{n}^{0} \vee \cdots \vee e_{n}^{n-1}$ and $*\left(e_{n}^{0} \vee \cdots \vee e_{n}^{n-1}\right)=1$,

(iii) $*(*(A))=(-1)^{k(n-k)} A$ if $A$ is of grade $k$.

The Hodge star realizes the duality between the meet and the join products [1. Hence, the following definition of the meet product, denoted $\wedge$, can be adopted:

$$
*(A \vee B)=*(A) \wedge *(B) \quad \text { and } \quad *(A \wedge B)=*(A) \vee *(B) .
$$

Thus, in the algebra $G_{3}$, the meet product can be defined over the basis elements by the table

\begin{tabular}{c|c|c|c|c|c|c|c|c|c|}
$\wedge$ & 1 & $e_{3}^{0}$ & $e_{3}^{1}$ & $e_{3}^{2}$ & $e_{3}^{0} \vee e_{3}^{1}$ & $e_{3}^{0} \vee e_{3}^{2}$ & $e_{3}^{1} \vee e_{3}^{2}$ & $e_{3}^{0} \vee e_{3}^{1} \vee e_{3}^{2}$ \\
\hline 1 & 0 & 0 & 0 & 0 & 0 & 0 & 0 & 1 \\
$e_{3}^{0}$ & 0 & 0 & 0 & 0 & 0 & 0 & 1 & $e_{3}^{0}$ \\
$e_{3}^{1}$ & 0 & 0 & 0 & 0 & 0 & -1 & 0 & $e_{3}^{1}$ \\
$e_{3}^{2}$ & 0 & 0 & 0 & 0 & 1 & 0 & 0 & $e_{3}^{2}$ \\
$e_{3}^{0} \vee e_{3}^{1}$ & 0 & 0 & 0 & 1 & 0 & $e_{3}^{0}$ & $e_{3}^{1}$ & $e_{3}^{0} \vee e_{3}^{1}$ \\
$e_{3}^{0} \vee e_{3}^{2}$ & 0 & 0 & -1 & 0 & $-e_{3}^{0}$ & 0 & $e_{3}^{2}$ & $e_{3}^{0} \vee e_{3}^{2}$ \\
$e_{3}^{1} \vee e_{3}^{2}$ & 0 & 1 & 0 & 0 & $-e_{3}^{1}$ & $-e_{3}^{2}$ & 0 & $e_{3}^{1} \vee e_{3}^{2}$ \\
$e_{3}^{0} \vee e_{3}^{1} \vee e_{3}^{2}$ & 1 & $e_{3}^{0}$ & $e_{3}^{1}$ & $e_{3}^{1}$ & $e_{3}^{0} \vee e_{3}^{1}$ & $e_{3}^{0} \vee e_{3}^{2}$ & $e_{3}^{1} \vee e_{3}^{2}$ & $e_{3}^{0} \vee e_{3}^{1} \vee e_{3}^{2}$
\end{tabular}

\section{Data-structures}

The programming language of $\mathrm{CoQ}$ proof assistant [3] is a functional language with dependent types. It is then particularly suitable for the development of abstract algebra. In order to have a generic formalization, our development is parametrized by an abstract field $K$ and its usual operations: 


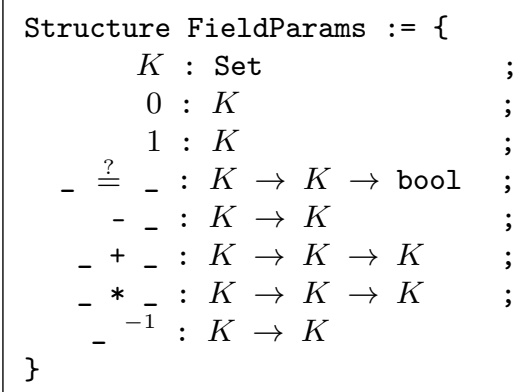

Note that even if every type in Coq is equipped with a propositional equality, i.e. for two elements $x$ and $y$ in $\mathrm{K}$ the proposition $x=y$ expresses that they are equal with respect to Leibnitz equality, we have an explicit equality test $x \stackrel{?}{=} y$ that lets us decide on this equality. This capability is crucial when defining algorithms over elements of $K$. Along with this parametric definition of $K$, there is an associated set of axioms that gives the usual basic properties of the operations (associativity, commutativity, distributivity and neutral elements).

From now on, all our definitions are taking this field $\mathrm{K}$ and another parameter $n$ for the dimension as parameters. They follow the same pattern: they are defined recursively on the dimension $n$. For a data-structure $D$, this means that its version $D_{n+1}$ for the $n+1$ dimension is going to be expressed in term of $D_{n}$. In this work, only the primitive pairing construct of COQ is used: if $a_{1}$ is of type $T_{1}$ and $a_{2}$ of type $T_{2},\left(a_{1}, a_{2}\right)$ is of type $T_{1} \times T_{2}$.

\subsection{Representing the vector space $K^{n}$}

As a first example, here is how the vectors of $K^{n}$ are defined for $n \neq 0$ :

Definition $K_{n}$ := if $n=1$ then $K$ else $K \times K_{n-1}$.

Compare to traditional programming where vectors would be represented as arrays, here we use recursion and pairing to mimic this data-structure. The typ $\AA^{3} K_{1}$ is equivalent to $K, K_{2}$ to $K \times K$ and $K_{3}$ to $K \times(K \times K)$ and an element of $K_{3}$ is represented by $\left(x_{1},\left(x_{2}, x_{3}\right)\right)$.

Operations on this data-structure are also defined recursively. For example, addition of two vectors of dimension $n$ is defined recursively as follows:

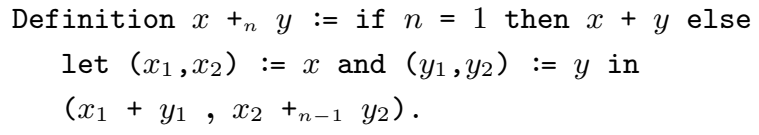

${ }^{3}$ The exponent $n$ is changed into an index for notational purpose. 
If the parameter $n$ is one, the two elements belong to $K$ so we can add them using the addition on $K$, otherwise each element can be decomposed into an element of $K$ and an element of one dimension less and the resulting pair can be composed by adding the elements of $K$ on the left and using a recursive call on the right. To end the vector space structure, scalar multiplication can be defined in a similar way:

Definition $k \cdot{ }_{n}$ y := if $n=1$ then $k * x$ else

let $\left(x_{1}, x_{2}\right):=x$ in $\left(k * x_{1}, k \cdot_{n-1} x_{2}\right)$.

\subsection{Representing the algebra $G_{n}$}

Representing elements of $G_{n}$, the Grassmann-Cayley of dimension $n$, follows exactly the same schema. This time, instead of a linear data-structure, binary trees are used:

Definition $G_{n}:=$ if $n=0$ then $K$ else $G_{n-1}$ x $G_{n-1}$.

The type $G_{0}$ is equivalent to $K, G_{1}$ to $K \times K, G_{2}$ to $(K \times K) \times(K \times K)$. Elements of $G_{n}$ are binary trees of height $n$. They have $2^{n}$ leaves. This corresponds to the fact that $G_{n}$ is a vector space of dimension $2^{n}$.

The sum and the scalar multiplication for the vector space structure are defined recursively over the dimension as follows:

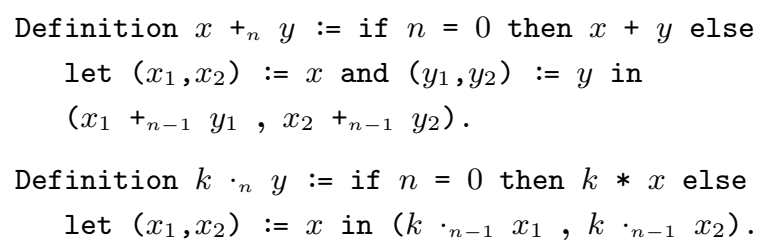

The equality test is defined in the same way:

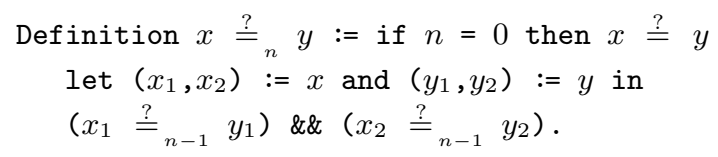

In this definition the operator \&\& is a special notation used in this paper for the logical and to avoid confusion with the meet product.

Figure 1 explains how the basis components of $G_{n}$ are mapped to the binary structure. The leaves contain the coefficients. For example, the grade 2 element of $G_{3}$,

$$
\text { 2. }\left(e_{3}^{0} \vee e_{3}^{1}\right)+3 .\left(e_{3}^{1} \vee e_{3}^{2}\right)
$$




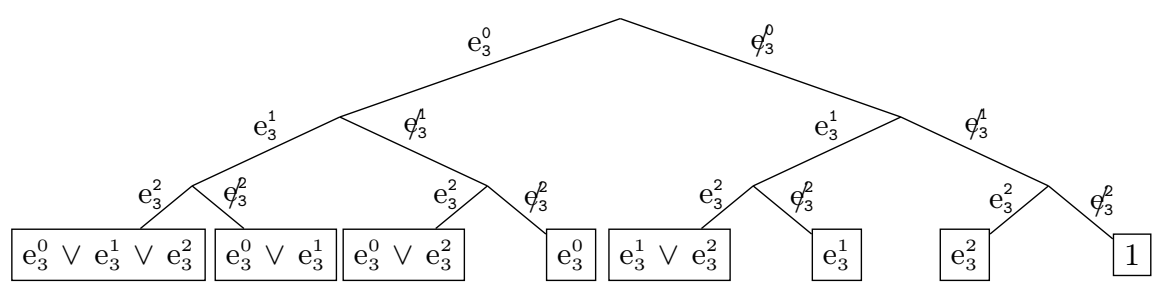

Fig. 1. Mapping of the multi-vector coefficients to the leaves of the binary tree.

is represented as $(((0,2),(0,0)),((3,0),(0,0)))$ and the multi-vector of $G_{3}$,

$$
2 .\left(e_{3}^{0} \vee e_{3}^{2}\right)+3 . e_{3}^{1}+4.1
$$

is represented as $(((0,0),(2,0)),((0,3),(0,4)))$. Here the sign sum indicates that an element of $G_{n}$ is a linear combination of the basis components.

For a tree of height $n$, at the level $i$, a move toward the left child inserts the basis element $e_{n}^{i}$ into the join product, while a move toward the right child insures that this element is not present. Then, on a path from the root of the tree to a leaf a move toward the left child increases the grade by one, while a move toward the right child leaves it unchanged. Hence, the left-most leaf of a tree of height $n$ contains the grade $n$ coefficient of an element of $G_{n}$ while the right-most leaf contains the coefficient of the grade 0 part.

This binary tree structure also allows to increase the dimension of an element $x$ of $G_{n}$ by injecting it to $G_{n+1}$. This is done with the function $\operatorname{inj}_{G_{n}}$ by simply pairing the binary tree of height $n$ with all its leaves containing 0 , denoted $0_{n}$, and $x$ :

Definition $\operatorname{inj}_{G_{n}} x:=\left(0_{n}, \mathrm{x}\right)$.

This operation does not change the grade of $x$, but it shifts the basis components. The basis component $e_{n}^{i} \vee \cdots \vee e_{n}^{i+k}$ is mapped to the basis component $e_{n+1}^{i+1} \vee$ $\cdots \vee e_{n+1}^{i+k+1}$.

From the mapping of the multi-vector coefficients and the injection function, the pairing of two elements $x$ and $y$ of $G_{n}$ can be interpreted in terms of an element of $G_{n+1}$. The pair $(x, y)$ represents the element

$$
e_{n+1}^{0} \vee \operatorname{inj}_{G_{n}} x+{ }_{n+1} \operatorname{inj}_{G_{n}} y .
$$

This means that pairing two elements $x$ and $y$ inserts the basis component $e_{n+1}^{0}$ into the shifted basis component of $x$. This puts all the coefficients of $x$ into the left part of the tree.

Now, the field $K$ is injected into the binary tree structure representing $G_{n}$ with the following functions: 


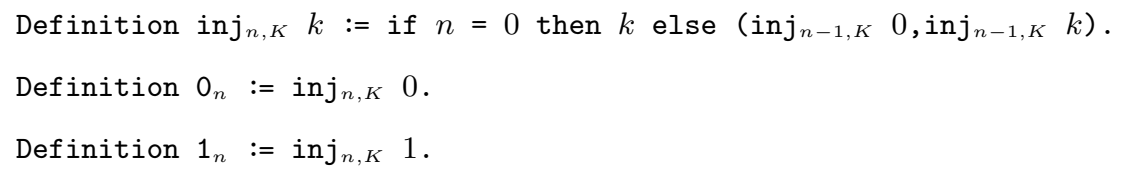

Hence, the tree representing zero has all its leaves set to 0 , while the tree representing one has only its right-most leaf set to 1 .

Using the injection of $K$ into $G_{n}$, we can define the injection of the elements of $K_{n}$ into $G_{n}$ :

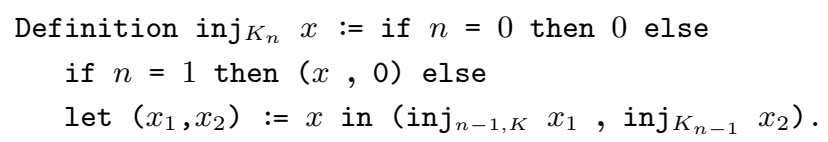

Note that, as $K_{0}$ is not defined, a special case is introduced for $n=0$ sending any element to zero. Let us take a concrete example to explain how this injection works. An element of $K_{3}$ is represented by a triplet $\left(x_{1},\left(x_{2}, x_{3}\right)\right)$. The element $\left(\left((0,0),\left(0, x_{1}\right)\right),\left(\left(0, x_{2}\right),\left(x_{3}, 0\right)\right)\right)$ of $G_{3}$ is its image by the injection. For an element of $K_{n}$, the coordinates $x_{i}$ are the coefficients of the basis elements $e_{n}^{i}$

We can also directly exhibit a base $\left\{e_{n}^{0}, e_{n}^{1}, \ldots, e_{n}^{n-1}\right\}$ for the vectors of $G_{n}$, i.e. the image by the injection $\operatorname{inj}_{K_{n}}$ of the base of $K_{n}$ induced by the coordinates. Again, this is defined recursively:

Definition $\mathrm{e}_{n}^{i}:=$ if $n=0$ then $1_{n}$ else

if $i=0$ then $\left(1_{n-1}, 0_{n-1}\right)$ else $\left(0_{n-1}, \mathrm{e}_{n-1}^{i-1}\right)$.

If we go back to the relation between $K_{3}$ and $G_{3}$, the base of $K_{3}$ induced by the coordinates is $\{(1,0,0),(0,1,0),(0,0,1)\}$. Then, we have

$$
\begin{aligned}
& \mathrm{e}_{3}^{0}=(((0,0),(0,1)),((0,0),(0,0))) \text { which corresponds to }(1,0,0), \\
& \mathrm{e}_{3}^{1}=(((0,0),(0,0)),((0,1),(0,0))) \text { which corresponds to }(0,1,0), \\
& \mathrm{e}_{3}^{2}=(((0,0),(0,0)),((0,0),(1,0))) \text { which corresponds to }(0,0,1) .
\end{aligned}
$$

When they are injected respectively into $G_{n}$ and $G_{n+1}$, the basis elements of $K_{n}$ and $K_{n+1}$ are related by $\mathrm{e}_{n+1}^{i+1}=\operatorname{inj}_{G_{n}} \mathrm{e}_{n}^{i}$. Hence, in terms of trees, the basis element $e_{n}^{i}$ is the right child of the basis element $e_{n+1}^{i+1}$. In the previous example, we can observe the left zero tree in the representation of $e_{3}^{2}$ and $e_{3}^{1}$ indicating that $e_{3}^{2}=\operatorname{inj}_{G_{2}} e_{2}^{1}$ and $e_{3}^{1}=\operatorname{inj}_{G_{2}} e_{2}^{0}$. This is coherent with interpretation of the pairing of two elements of $G_{n}$ (see the relation (2)).

At the moment, from the point of view of the properties that can be formally proved, only the usual properties of vector space for $K_{n}$ and $G_{n}$ and the properties of morphism of the different injections can be derived. 


\subsection{Join product}

The next step is to define the join product as a binary tree operation. To explain the definition, we use equation (2) and the mandatory properties expressed by the axioms (1) in section 2.2 .

The idea is to define the join product recursively over the dimension. To do so, we decompose the product $x \vee_{n} y$ in terms of pairing and using the relation (2), we obtain:

$$
\begin{aligned}
x \vee_{n} y & =\left(x_{1}, x_{2}\right) \vee_{n}\left(y_{1}, y_{2}\right) \\
& =\left(e_{n}^{0} \vee_{n} \operatorname{inj}_{G_{n-1}} x_{1}+{ }_{n} \operatorname{inj}_{G_{n-1}} x_{2}\right) \vee_{n}\left(e_{n}^{0} \vee_{n} \operatorname{inj}_{G_{n-1}} y_{1}+{ }_{n} \operatorname{inj}_{G_{n-1}} y_{2}\right)
\end{aligned}
$$

Then, using the axioms (1), we obtain:

$$
\begin{aligned}
\left(x_{1}, x_{2}\right) \vee_{n}\left(y_{1}, y_{2}\right) & =e_{n}^{0} \vee_{n} \operatorname{inj}_{G_{n-1}} x_{1} \vee_{n} \operatorname{inj}_{G_{n-1}} y_{2} \\
& +{ }_{n} \operatorname{inj}_{G_{n-1}} x_{2} \vee_{n} e_{n}^{0} \vee_{n} \operatorname{inj}_{G_{n-1}} y_{1} \\
& +{ }_{n} \operatorname{inj}_{G_{n-1}} x_{2} \vee_{n} \operatorname{inj}_{G_{n-1}} y_{2} .
\end{aligned}
$$

In the second term in the sum of the right part of this latter expression, the factor $e_{n}^{0}$ needs to be commuted with $\operatorname{inj}_{G_{n-1}} x_{2}$ in order to be able to factorize the expression with $e_{n}^{0}$ and to get an expression that corresponds to a pairing.

However, the join product is anti commutative and we must pay attention to sign changes into the factors. For example, if $x$ is an homogeneous element of grade $k$, we have $\mathrm{e}_{n}^{i} \vee x=(-1)^{k} \cdot x \vee \mathrm{e}_{n}^{i}$. We want to generalize this property and have a conjugate function, noted $\bar{x}$, such that $\mathrm{e}_{n}^{i} \vee x=\bar{x} \vee \mathrm{e}_{n}^{i}$ for all $x$ in $G_{n}$. Here is the definition of such a function:

Definition $\bar{x}^{\mathrm{n}}:=$ if $n=0$ then $x$ else

let $\left(x_{1}, x_{2}\right):=x$ in $\left(-\bar{x}_{1}^{n-1},{\overline{x_{2}}}^{\mathrm{n}-1}\right)$.

Now expression (3) can be rewritten as:

$$
\begin{aligned}
\left(x_{1}, x_{2}\right) \vee_{n}\left(y_{1}, y_{2}\right)= & e_{n}^{0} \vee_{n}\left(\operatorname{inj}_{G_{n-1}} x_{1} \vee_{n} \operatorname{inj}_{G_{n-1}} y_{2}\right. \\
& \left.+{ }_{n} \operatorname{inj}_{G_{n-1}} x_{2}{ }^{n} \vee_{n} \operatorname{inj}_{G_{n-1}} y_{1}\right) \\
+ & { }_{n} \operatorname{inj}_{G_{n-1}} x_{2} \vee_{n} \operatorname{inj}_{G_{n-1}} y_{2} .
\end{aligned}
$$

Using the definition of the injection $\operatorname{inj}_{G}$, we get:

$$
\begin{aligned}
\left(x_{1}, x_{2}\right) \vee_{n}\left(y_{1}, y_{2}\right) & =e_{n}^{0} \vee_{n}\left(\operatorname{inj}_{G_{n-1}}\left(x_{1} \vee_{n-1} y_{2}+_{n-1}{\overline{x_{2}}}^{n-1} \vee_{n-1} y_{1}\right)\right) \\
& { }_{n} \operatorname{inj}_{G_{n-1}}\left(x_{2} \vee_{n-1} y_{2}\right) .
\end{aligned}
$$

This leads to the following recursive definition of the join product:

$$
\begin{aligned}
& \text { Definition } x \vee_{n} y:=\text { if } n=0 \text { then } x * y \text { else } \\
& \text { let }\left(x_{1}, x_{2}\right):=x \text { and }\left(y_{1}, y_{2}\right):=y \text { in } \\
& \left(x_{1} \vee_{n-1} y_{2}{ }^{{ }_{n-1}}{\overline{x_{2}}}^{\mathrm{n}-1} \vee_{n-1} y_{1}, x_{2} \vee_{n-1} y_{2}\right)
\end{aligned}
$$

From this definition, we have proved formally that this join product verifies its basic properties (associativity, bilinearity and anti commutativity) defined by the axioms (1). 


\subsection{Meet Product}

In order to define the meet product, we follow exactly the same path than for the join product. We generalize the fact that, for an homogeneous element $x$ of grade $k$ of $G_{n}$, we have $\mathrm{e}_{n}^{i} \wedge x=(-1)^{(n-k)} \cdot x \wedge \mathrm{e}_{n}^{i}$ and define a dual version of the conjugate function, noted $\bar{x}^{d}$, such that $\mathrm{e}_{n}^{i} \wedge x=\bar{x}^{d} \wedge \mathrm{e}_{n}^{i}$.

Definition $\bar{x}^{d n}:=$ if $n=0$ then $x$ else

let $\left(x_{1}, x_{2}\right):=x$ in $\left(\bar{x}_{1}^{d n-1},-\bar{x}^{d n-1}\right)$.

Again, with this auxiliary function, the meet product can be defined recursively as follows:

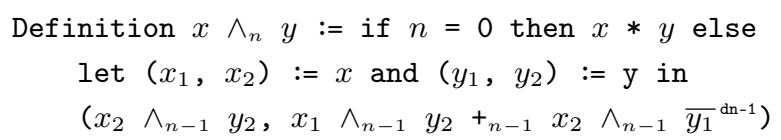

Note that our recursive approach avoids the use of bracket algebra to define the meet product so that our formalization works internally and independently from the bracket algebra framework. As for the join product, it is quite direct to derive the basic properties of the meet product formally from its definition.

\subsection{Duality}

The Hodge star operator presented in section 2.3 is also defined recursively over the dimension as follows:

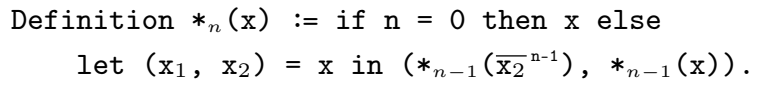

Note that in our representation, upto sign flips, the Hodge star just reverses the leaves of the binary tree. For example in $G_{3}$, the dual of the element

$$
\left(\left(\left(x_{1}, x_{2}\right),\left(x_{3}, x_{4}\right)\right),\left(\left(x_{5}, x_{6}\right),\left(x_{7}, x_{8}\right)\right)\right)
$$

is the reverse element with two sign flips

$$
\left(\left(\left(x_{8}, x_{7}\right),\left(-x_{6}, x_{5}\right)\right),\left(\left(x_{4},-x_{3}\right),\left(x_{2}, x_{1}\right)\right)\right) .
$$

Because of these sign flips, the Hodge star is not an involution. For homogeneous elements, the following theorem (corresponding to property (iii) in section 2.3 is proved into our formalization using the defined Hodge star:

Lemma dual_invo: $\forall n k v$, if $\operatorname{hom}_{n}^{k} v$ then $*_{n}\left(*_{n}(v)\right)=(-1)^{k(n-k)} \cdot_{n} v$. 
where hom ${ }_{n}^{k}$ tests if an element is homogeneous and is defined as follows:

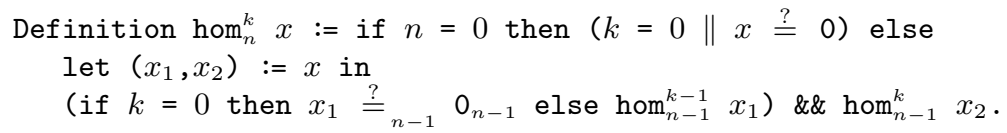

As previously, the notation $\|$ is a special notation for the logical or to avoid confusion with the join product.

Due to our choice of the underlying vector space basis, the Hodge star implements the duality between the join product and the meet product (see section 2.3 and reference [1]). Then the following theorem are proved within our CoQ formalization:

Lemma dual_prod: $\forall n \quad v_{1} v_{2}, *_{n}\left(\begin{array}{lll}v_{1} & \vee_{n} & v_{2}\end{array}\right)=*_{n}\left(v_{1}\right) \wedge_{n} *_{n}\left(v_{2}\right)$.

Lemma dual_dprod: $\left.\forall n \begin{array}{llllll}v_{1} & v_{2}, *_{n}\left(v_{1}\right. & \wedge_{n} & v_{2}\end{array}\right)=*_{n}\left(v_{1}\right) \vee_{n} *_{n}\left(v_{2}\right)$.

This proves that the join product and the meet product are correctly defined.

At this point, Grassmann-Cayley algebra could already be considered as formalized in the CoQ proof assistant.

\section{Theorem Proving in Projective Geometry}

In this section, we first show how we can use our formalization of GrassmannCayley algebra to model the geometry of incidence. Then, in a second step, we show how proofs in this setting can be fully automatized within CoQ.

\subsection{Modeling the Geometry of Incidence}

Now that we have Grassmann-Cayley algebra in CoQ, we can use it to represent theorems in projective geometry. All this is standard and can be found by example in [18] or in [15] chapter 3. We just explain how this has been instantiated to our formalization. We work over an arbitrary field $K$ and restrict ourselves to $G_{3}$. We take a conservative approach and consider only non-degenerated configurations for constructed points. In this setting, points are vectors, so in our case we are going to use our injection from $K_{3}$ to $G_{3}$ :

Definition point $K:=K_{3}$.

To define the fact that a point $p_{1}$ is the intersection of the line composed of $p_{2}$ and $p_{3}$ and the line composed of $p_{4}$ and $p_{5}$, we simply implement it by saying that using the join to create the line and the meet to perform the intersection:

Definition $p_{1}$ is the intersection of $\left[p_{2}, p_{3}\right]$ and $\left[p_{4}, p_{5}\right]:=$

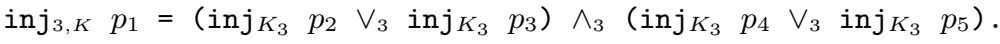


Note that the equality imposes the meet product to be a point, so the lines to be defined and intersecting.

To define the fact that a point $p_{1}$ is on the line composed of $p_{2}$ and $p_{3}$, we simply implement it by saying that the line is well-defined, i.e. the join product of $p_{2}$ and $p_{3}$ is not zero, and the joint product of the three points is zero:

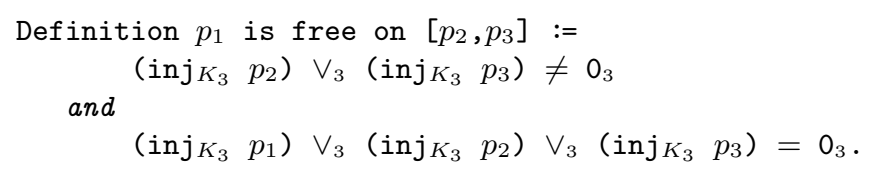

Finally, we consider the collinearity of three points and the concurrency of three lines:

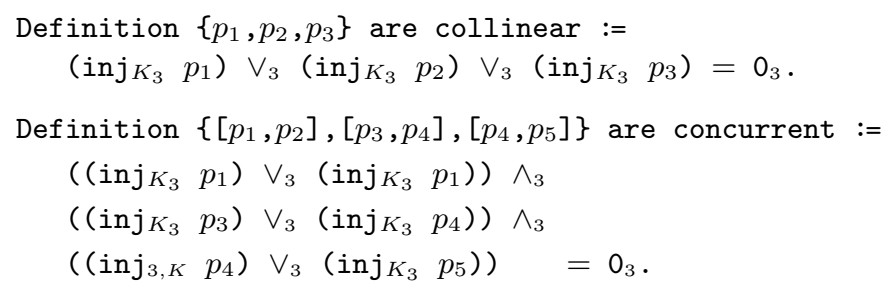

With these definitions, we can start stating some classic theorems of geometry of incidence. First, let us consider Pappus' theorem:

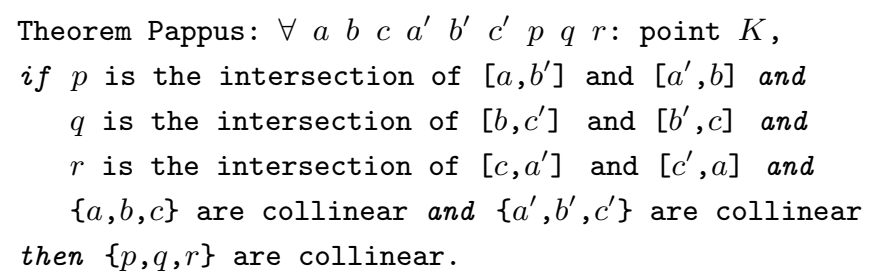

Introducing the universal quantification and eliminating the points $p, q$ and $r$, we are left with proving that

$$
\begin{aligned}
& \text { if } a \vee b \vee c=0 \text { and } a^{\prime} \vee b^{\prime} \vee c^{\prime}=0 \text { then } \\
& \left(a \vee b^{\prime} \wedge a^{\prime} \vee b\right) \vee\left(b \vee c^{\prime} \wedge b^{\prime} \vee c\right) \vee\left(b \vee c^{\prime} \wedge b^{\prime} \vee c\right)=0
\end{aligned}
$$

Remaining inside the algebra and applying the basic properties it is possible to prove this statement interactively in CoQ. For this, we have followed of the proof given in [7. This requires 10 interactions where the prover is guided in order to apply the symbolic manipulations that leads to the proof.

A more involved proof is Desargues' theorem. It can be stated as:

\footnotetext{
${ }^{4}$ We voluntarily omit the injections and the indices to make the expression more legible.
} 


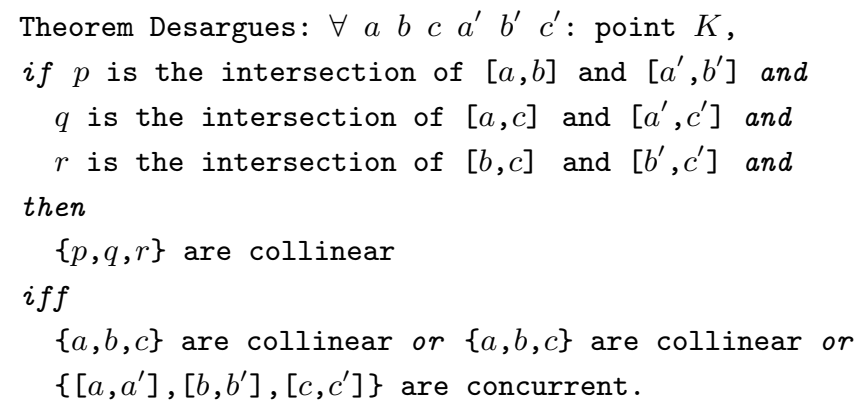

Again, introducing the universal quantification and eliminating the points $p, q$ and $r$, we are left with proving that:

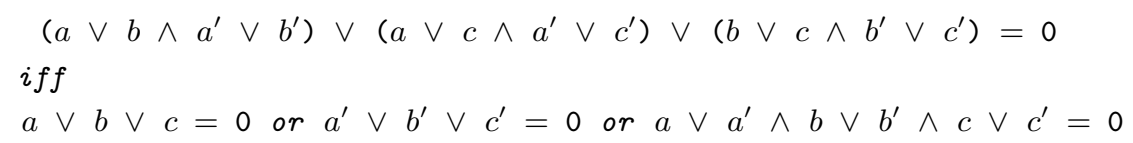

In order to prove this interactively, this time we have followed the paper proof given in [1]. The proof is more intricate and has required 60 interactions with the prover.

\subsection{Automating Proofs}

Proving the last two theorems is very satisfying because it shows that our algebra can be manipulated symbolically within CoQ but clearly we are at the limit of what is bearable for a user to prove interactively. So, the next step is to automate the proof of such theorems. For this, we are going to introduce bracket algebra and follow the path of [11].

Bracket algebra and its relation with Grassmann-Cayley is a well-known topic [61]. Here, we are just going to explain how it has been introduced in our setting. For the moment, this has only been implemented for $G_{3}$ but we believe that this could be easy generalised to $G_{n}$ for an arbitrarily $n$. In the following, in order to increase legibility we will systematically omit the indices and the injections, so for example $\left(\operatorname{inj}_{K_{3}} p_{1} \vee_{3} \operatorname{inj}_{K_{3}} p_{2}\right)$ will be noted $\left(p_{1} \vee p_{2}\right)$ only. A bracket is a function that takes three points and returns an element of our field $K$. Its definition is the following:

Definition $\left[p_{1}, p_{2}, p_{3}\right]:=\mathrm{dC}\left(p_{1} \vee p_{2} \vee p_{3}\right)$

where $\mathrm{dC}$ stands for the dual of the constant component, i.e the left-most leaf of the tree-structure given in Figure 1 of page 7. The usual relations between the bracket, the join product and the meet product in $G_{3}$ are derived. 


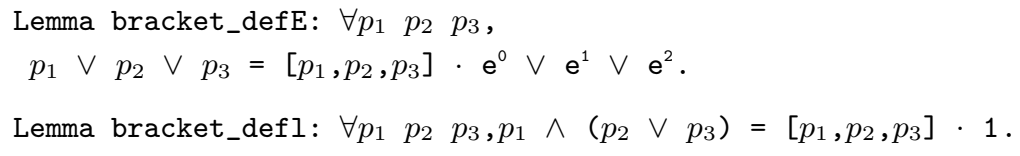

We have also formally proved that it behaves as a determinant:

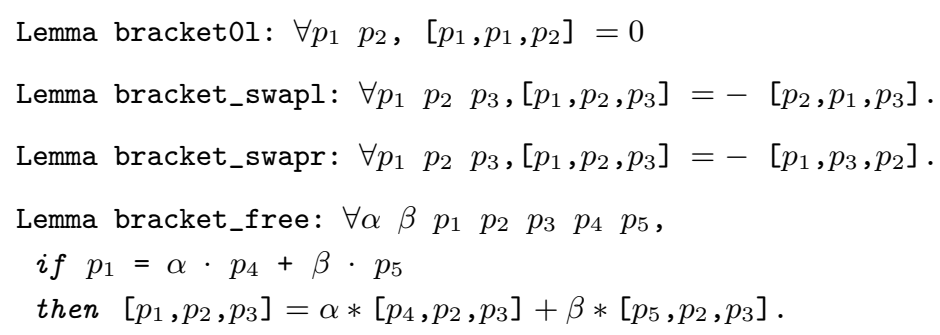

In order to automate as described in 11, we are going to restrict ourselves to a specific skeleton of proofs. The goals we are going to be able to prove automatically have the following shape:

$$
\forall p_{1} p_{2} \ldots p_{m} \text {, if } H_{1} \text { and } \ldots H_{n} \text { then }\left\{p_{i}, p_{j}, p_{k}\right\} \text { are collinear }
$$

where the $H_{i}$ s are either the construction of a free point on a line

$$
p_{j} \text { is free on }\left[p_{r}, p_{s}\right]
$$

or the construction of an intersection

$$
p_{j} \text { is the intersection of }\left[p_{r}, p_{s}\right] \text { and }\left[p_{t}, p_{u}\right] \text {. }
$$

How does the automatic procedure proceed? As the conclusion is a collinearity property, it can be turned into an equality to zero of a bracket expression by the following lemma that is a direct consequence of the lemma bracket_defE:

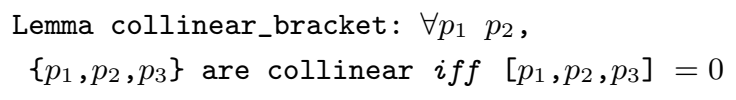

Then, the constructed points are progressively eliminated from the assumptions to obtain a bracket expression. Two lemmas are used corresponding to each construction. In the first case, the assumption is the construction of a free point on a line. The following lemma can be proved thanks to the conservative approach we observed:

Lemma online_def: $\forall p_{1} p_{2} p_{3}$, if $p_{1}$ is free on $\left[p_{2}, p_{3}\right]$ then $\exists \alpha \beta, p_{1}=\alpha \cdot p_{2}+\beta \cdot p_{3}$ 
Coupled with the lemma bracket_free, this lets us remove the free point from all bracket expressions. In the second case, the assumption is the construction of an intersection then the second rule ${ }^{5}$ given in [11] is used to remove the point:

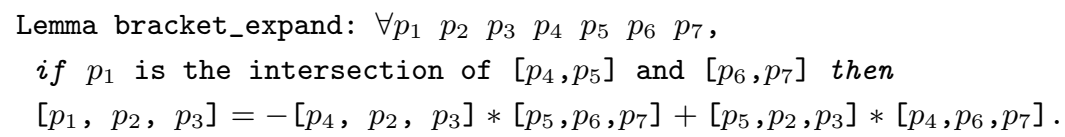

Once all the eliminations of constructed points have been performed, we get an expression that contains sums and products of bracket of initial points and the $\alpha \mathrm{s}$ and the $\beta$ s introduced by the eliminations of the free points. So for the theorem to be true generically, this expression must be equal to zero modulo Plücker relations (see [11). In order to simplify the obtained expression a contraction rule is used in [11]). In our setting it is stated as:

$$
\begin{aligned}
& \text { Lemma contraction_v0: } \forall p_{1} p_{2} p_{3} p_{4} p_{5}, \\
& {\left[p_{1}, p_{2}, p_{3}\right] *\left[p_{1}, p_{4}, p_{5}\right]-\left[p_{1}, p_{2}, p_{5}\right] *\left[p_{1}, p_{4}, p_{3}\right]=\left[p_{1}, p_{2}, p_{4}\right] *\left[p_{1}, p_{3}, p_{5}\right] .}
\end{aligned}
$$

Surprisingly applying this rule unrestrictively as a rewrite rule from left to right as described in [11] is very effective. However, it is not sufficient in our setting to prove all the given examples. To fix this problem, we implement a normalisation method that is very expensive but is known to be complete. This captures the remaining examples. The method is based on an implicit ordering of the initial points $p_{1}<p_{2}<\cdots<p_{i}$. Applying some permutation, brackets can always be ordered with respect to this order: $\left[p_{i}, p_{j}, p_{k}\right]$ with $p_{i}<p_{j}<p_{k}$.

The order can be lifted to brackets $\left[p_{i}, p_{j}, p_{k}\right] \leq\left[p_{i^{\prime}}, p_{j^{\prime}}, p_{k^{\prime}}\right]$ if $p_{i} \leq p_{i^{\prime}}$ and $p_{j} \leq p_{j^{\prime}}$ and $p_{k} \leq p_{k^{\prime}}$. The normalisation proceeds in trying to order the product of brackets from the smallest to the largest. For this, we consider the product of two brackets $\left[p_{i}, p_{j}, p_{k}\right] *\left[p_{i^{\prime}}, p_{j^{\prime}}, p_{k^{\prime}}\right]$. Without loss of generality, we can suppose that $p_{i} \leq p_{i^{\prime}}$. There are only two situations where this product is not ordered:

1. $p_{i}<p_{i^{\prime}}$ and $p_{j^{\prime}}<p_{j}$

2. $p_{i} \leq p_{i^{\prime}}$ and $p_{j}<p_{j^{\prime}}$ and $p_{k^{\prime}}<p_{k}$ (or equivalently $p_{i}<p_{i^{\prime}}$ and $p_{j} \leq p_{j^{\prime}}$ and $\left.p_{k^{\prime}}<p_{k}\right)$

The first rewrite rule takes care of the first case and assures that the resulting expression has every first two elements of brackets in a product properly ordered.

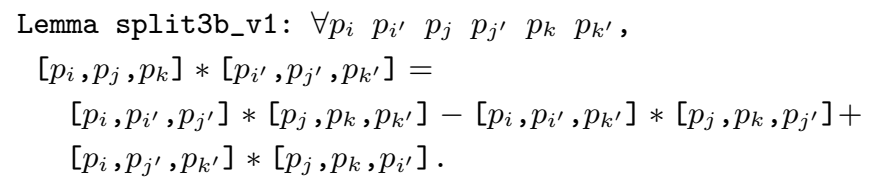

${ }^{5}$ This rule corresponds to the elimination of the area method 9 . 
The second rewrite rule takes care of the second case and insures that the resulting products of brackets are all properly ordered.

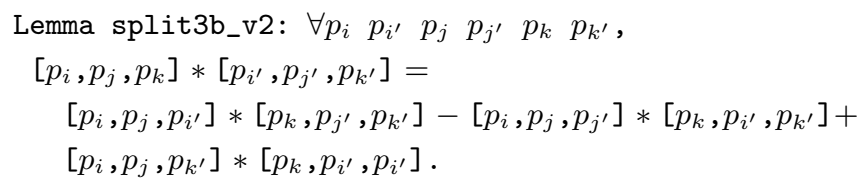

\section{Conclusion}

We have described how our formalization of Grassmann-Cayley algebra has been achieved. It is a generic one: it is parametrized both by the underlying field $K$ and by the dimension $n$. A snapshot of the formalization with a complete zipped archive is available at

$$
\text { http://www-sop.inria.fr/marelle/GeometricAlgebra. }
$$

Recursive definitions have played a central role in this formalization. Elements of the algebra are represented as binary trees. With this representation, operations like the meet, the join and the duality can be described as recursive functions in a very direct way. The nice thing about defining these operations in a proof assistant like CoQ is that not only can we compute with them like in any programming language but also we can reason about them. This lets us derive all the standard properties of Cayley-Grassmann operations. Our implementation is then verified: we have a certified computational model of Grassmann-Cayley algebra.

One of the most satisfying part of this formalization is, without a doubt, the instantiation that has been done in order to prove Pappus' and Desargues' theorem as proposed in [7] and [1. We have been capable of justifying formally every step of the paper proofs. Moreover, the method that automatically proves projective geometric theorems proposed in [11] has also be translated successfully into our formalization. An efficient CoQ tactic has been developed. This makes us very confident in the potential of our formalization.

In addition to the formalization of the Grassmann-Cayley algebra basics properties, we have also considered other useful operations such as contraction $\langle\phi, v\rangle$ of a linear form $\phi$ on a vector $v$ and factorisation have also been formalized.

As already mentioned in the introduction, we are very interested in studying the links with other formalized approaches of incidence geometry such as those based over ranks [14 13].

Finally, we also plan to develop our formalization to capture the powerful framework of the geometric algebra [8]5]

\section{References}

1. Barnabei, M., Brini, A., Rota, G.C.: On the Exterior Calculus of Invariant Theory. Journal of Algebra 96, 120-160 (1985) 
2. Bertot, Y., Castéran, P.: Interactive Theorem Proving and Program Development, Coq'Art: The Calculus of Inductive Constructions. Springer (2004)

3. Coq development team: The Coq Proof Assistant Reference Manual, Version 8.2. LogiCal Project (2008), http://coq.inria.fr

4. Crapo, H., Richter-Gebert, J.: Automatic proving of geometric theorems. In: White [16], pp. 167-196

5. Dorst, L., Fontijne, D., Mann, S.: Geometric Algebra for Computer Science: An Object Oriented Approach to Geometry. Morgan Kauffmann Publishers (2007)

6. Doubilet, P., Rota, G.C., Stein, J.: On the foundations of combinatorial theory. IX. Combinatorial methods in invariant theory. Studies in Applied Mathematics $53,185-216(1974)$

7. Hawrylycz, M.: A geometric identity for Pappus' theorem. Proceedings of the National Academy of Sciences U.S.A. 91(8), 2909 (1994)

8. Hestenes, D., Sobczyk, G.: Clifford Algebra to Geometric Calculus: A Unified Language for Mathematics and Physics, Fundamental Theories of Physics, vol. 5. Kluwer Academic Publishers (1984)

9. Janicic, P., Narboux, J., Quaresma, P.: The Area Method : a Recapitulation. Journal of Automated Reasoning (2010), published online

10. Li, H.: Algebraic Representation, Elimination and Expansion in Automated Geometric Theorem Proving. In: ADG'02. LNAI, vol. 2930, pp. 106-123 (2002)

11. Li, H., Wu, Y.: Automated short proof generation for projective geometric theorems with Cayley and bracket algebras: I. Incidence geometry. Journal of Symbolic Computation 36(5), 717-762 (2003)

12. Magaud, N., Narboux, J., Schreck, P.: Formalizing Projective Plane Geometry in Coq. In: Proceedings of ADG'2008. pp. 1-20 (Sept 2008)

13. Magaud, N., Narboux, J., Schreck, P.: Formalizing Desargues' theorem in Coq using ranks. In: Proceedings of the ACM Symposium on Applied Computing SAC 2009. ACM, ACM Press (March 2009), http://lsiit.u-strasbg.fr/Publications/ 2009/MNS09

14. Michelucci, D., Schreck, P.: Incidence constraints: A combinatorial approach. International Journal of Computational Geometry \& Applications 16(5-6), 443-460 (2006)

15. Sturmfels, B.: Algorithms in Invariant Theory. Springer, New York (1993)

16. White, N.L. (ed.): Invariants Methods in Discrete and Computational Geometry. Kluwer, Dordrecht (1995)

17. White, N.L.: A tutorial on Grassmann-Cayley algebra. In: Invariants Methods in Discrete and Computational Geometry [16], pp. 93-106

18. White, N.L.: Geometric applications of the Grassmann-Cayley algebra. In: Handbook of discrete and computational geometry, pp. 881-892. CRC Press, Inc., Boca Raton, FL, USA (1997) 